\title{
Copeptin with high-sensitivity troponin at presentation is not inferior to serial troponin measurements for ruling out acute myocardial infarction
}

\author{
Kyung Su Kim ${ }^{1}$, Gil Joon Suh ${ }^{1,2 *}$, Sang Hoon Song ${ }^{3 *}$, Yoon Sun Jung ${ }^{1}$, \\ Taegyun Kim ${ }^{1}$, So Mi Shin ${ }^{1}$, Min Woo Kang ${ }^{1}$, Min Sung Lee ${ }^{1}$ \\ 'Department of Emergency Medicine, Seoul National University Hospital, Seoul, Korea \\ ${ }^{2}$ Department of Emergency Medicine, Seoul National University College of Medicine, Seoul, Korea \\ ${ }^{3}$ Department of Laboratory Medicine, Seoul National University Hospital, Seoul, Korea
}

Objective We aimed to compare the multi-marker strategy (copeptin and high-sensitivity cardiac troponin I [hs-cTnl]) with serial hs-cTnl measurements to rule out acute myocardial infarction (AMI) in patients with chest pain.

Methods This prospective observational study was performed in a single emergency department. To test the non-inferiority margin of 4\% in terms of negative predictive value (NPV) between the multi-marker strategy ( 0 hour) and serial hs-cTnl measurements ( 0 and 2 hours), 262 participants were required. Samples for copeptin and hs-cTnl assays were collected at presentation (0 hour) and after 2 hours. The measured biomarkers were considered abnormal when hs-cinl was $>26.2 \mathrm{ng} / \mathrm{L}$ and when copeptin was $>10 \mathrm{pmol} / \mathrm{L}$.

Results AMI was diagnosed in 28 patients (10.7\%). The NPV of the multi-marker strategy was $100 \%$ (160/160; 95\% confidence interval [Cl], 97.7\% to 100\%), which was not inferior to that of serial hs-cTnl measurements (201/201; 100\%; 95\% Cl, 98.2\% to 100\%). The sensitivity, specificity, and positive predictive value of the multi-marker strategy were $100 \%(95 \% \mathrm{Cl}, 87.7 \%$ to 100\%), $68.1 \%(95 \% \mathrm{Cl}, 61.7 \%$ to $74.0 \%)$, and $27.2 \%(95 \% \mathrm{Cl}, 18.9 \%$ to $36.8 \%)$, respectively. The sensitivity, specificity, and positive predictive value of serial hs-cTnl measurements were $100 \%$ (95\% Cl, $87.7 \%$ to $100 \%)$, $85.5 \%$ (95\% Cl, 80.4\% to $89.8 \%$ ), and $45.2 \%(95 \% \mathrm{Cl}, 32.5 \%$ to $58.3 \%)$, respectively.

Conclusion The multi-marker strategy (copeptin and hs-cTnl measurement) was not inferior to serial hs-cTnl measurements in terms of NPV for AMI diagnosis, with a sensitivity and NPV of $100 \%$. Copeptin may help in the early rule-out of AMI in patients with chest pain.

Keywords Myocardial infarction; Troponin I; Biomarkers
eISSN: 2383-4625

Received: 18 February 2019

Revised: 1 April 2019

Accepted: 8 April 2019

*Correspondence to: Gil Joon Suh Department of Emergency Medicine, Seoul National University Hospital, 101 Daehak-ro, Jongno-gu, Seoul 03080, Korea

E-mail: suhgil@snu.ac.kr ORCID

https://orcid.org/0000-0001-5163-2217

*Correspondence to: Sang Hoon Song Department of Laboratory Medicine, Seoul National University Hospital, 101 Daehak-ro, Jongno-gu,

Seoul 03080, Korea

E-mail address: cloak21@snu.ac.kr ORCID

https://orcid.org/0000-0002-5084-1137

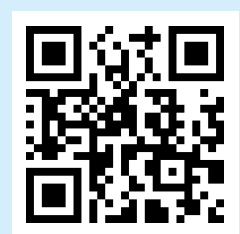

How to cite this article:

Kim KS, Suh GJ, Song SH, Jung YS, Kim T, Shin SM, Kang MW, Lee MS. Copeptin with high-sensitivity troponin at presentation is not inferior to serial troponin measurements for ruling out acute myocardial infarction. Clin Exp Emerg Med 2020;7(1):35-42.

This is an Open Access article distributed under the terms of the Creative Commons Attribution Non-Commercial License (http:// creativecommons.org/licenses/by-nc/4.0/). 


Capsule
$\begin{aligned} & \text { What is already known } \\ & \text { Serial high-sensitivity cardiac troponin measurements are essential to diagnose acute myocardial infarction. Recent } \\ & \text { studies have investigated the role of copeptin, the C-terminal part of arginine vasopressin, for the multi-marker strate- } \\ & \text { gy. However, high-sensitivity cardiac troponin assays were not used, and only additional diagnostic performance of co- } \\ & \text { peptin was examined in most studies. } \\ & \text { What is new in the current study } \\ & \text { The combined measurement of copeptin and high-sensitivity cardiac troponins showed 100\% (87.7\% to 100\%) sensi- } \\ & \text { tivity and 100\% (97.7\% to 100\%) negative predictive value for acute myocardial infarction. Moreover, the negative } \\ & \text { predictive value of the multi-marker strategy was not inferior to that of serial high-sensitivity cardiac troponin assays. }\end{aligned}$

\section{INTRODUCTION}

Acute myocardial infarction (AMI) is a leading cause of death worldwide, and chest pain is one of the most common complaints in the emergency department (ED). ${ }^{1}$ Although ST-elevation myocardial infarction (STEMI) can be identified using electrocardiography (ECG), the diagnosis of non-ST-elevation myocardial infarction (NSTEMI) is challenging. ${ }^{2}$ Cardiac troponins (cTns) are essential for NSTEMI diagnosis because of their high sensitivity and specificity. However, even in the era of high-sensitivity cardiac troponins (hs-cTns), serial measurements are mandatory because of the "troponin-blind interval."

Arginine vasopressin (AVP) plays an important role in maintaining hemodynamic stability through its antidiuretic and vasoconstriction effects in stressful conditions including AMI. ${ }^{3}$ However, the utility of AVP as a biomarker is limited because AVP is a small 9-amino acid peptide, which is mostly bound to platelets, and its plasma half-life is only 24 minutes. ${ }^{4}$ By contrast, copeptin is a 39 -amino acid peptide synthesized in the hypothalamus as the C-terminal part of the AVP prohormone. It is released in equimolar amounts with AVP and remains stable in plasma. ${ }^{5}$ This makes copeptin a surrogate marker for AVP in clinical conditions with high endogenous stress such as sepsis and AMI. ${ }^{6}$

Many studies have investigated the multi-marker strategy using cTn and copeptin at admission for the rapid rule-out of NSTEMl. ${ }^{7-13}$ However, they used conventional cTn assays or evaluated the additive diagnostic performance of copeptin to a single cTn assay. ${ }^{7-13}$ Furthermore, the additive diagnostic performance does not result in sufficient sensitivity to safely rule out NSTEMI in some studies. ${ }^{8,9,12}$

Thus, we aimed to compare the multi-marker strategy (copeptin and hs-cTn) at admission with serial hs-cTn measurements to rule out NSTEMI in ED patients with chest pain. We hypothesized that the negative predictive value (NPV) of the multi-marker strat- egy for NSTEMI is not inferior to that of serial hs-cTn measurements.

\section{METHODS}

\section{Study design}

This prospective observational study was performed in a single tertiary ED with an annual census of approximately 70,000 patients from January 2018 to August 2018. The study protocol was approved by the institutional review board (1709-126-890), and written informed consent was obtained from all participants.

\section{Study setting and population}

Patients who presented to the ED with complaints of chest pain were prospectively screened by a single researcher from 8:00 to 17:00 during weekdays. Patients were enrolled in the study if they did not fulfill the following exclusion criteria: age $\leq 18$ years, symptom onset to arrival time $\geq 12$ hours, STEMI on ECG, and refusal to participate.

\section{Study protocol}

The standardized protocol for patients with chest pain included history taking, physical examinations, ECG, serial high-sensitivity cardiac troponin I (hs-cTnl) measurements ( 0 and 2 hours), chest $X$-ray, and other laboratory examinations. ${ }^{14}$ If STEMI was suspected on ECG, then the interventional cardiology team was notified for immediate coronary angiography (CAG) and percutaneous coronary intervention. Cardiology consultation was performed for further evaluation and management when hs-cTnl was elevated. When serial hs-cTnl was within the normal limit, thrombolysis in myocardial infarction (TIMI) risk score was evaluated. Patients with a TIMI score of 2 or more require additional testing such as coronary computed tomography angiography to evaluate for coronary lesions. Patients with a TIMI score of 0 or 1 were consid- 
ered as low risk and thus could be discharged. The final discharge decision was at the discretion of the attending physician. An outpatient follow-up was recommended for all discharged patients.

\section{Measurements of clinical data}

The following patient information and clinical findings were obtained: age, sex, family history of coronary artery disease, hypertension, diabetes mellitus, hyperlipidemia, smoking, known coronary artery disease, aspirin use in the past 7 days, severe angina ( $\leq 2$ episodes in 24 hours), ST changes $\geq 0.5 \mathrm{~mm}$ on ECG, chest pain characterization (onset, location, duration, and radiation), TIMI risk scores, biomarkers (copeptin at 0 hour and hs-cTnl at 0 and 2 hours), additional diagnostic testing (coronary computed tomography angiography, treadmill test, cardiac scintigraphy, CAG, and percutaneous coronary intervention), ED disposition, and hospitalization outcome.

\section{Measurement of biomarkers}

Blood was drawn at admission (0 hour) and after 2 hours to measure hs-cTnl. Samples in serum separator tubes were transferred to the central laboratory and centrifuged, and the serum was then separated. ARCHITECT STAT High Sensitive Troponin-I assay (Abbott Laboratories, Abbott Park, IL, USA) was used for the analysis. The hs-cTnl assay has a 99th percentile concentration of $26.2 \mathrm{ng} /$ $L$ with a corresponding coefficient of variation of $4 \%$ and a limit of detection of $1.2 \mathrm{ng} / \mathrm{L}^{15}$

Copeptin was measured from the same blood sample drawn for hs-cTnl measurement after written informed consent was obtained. Thermo Scientific B.R.A.H.M.S Copeptin proAVP KRYPTOR assay (B.R.A.H.M.S. GmbH, Hennigsdorf/Berlin, Germany) was used. The assay has a 95th percentile concentration of $9.8 \mathrm{pmol} / \mathrm{L}$ with a corresponding intra-assay coefficient of variation of $<8 \%$ and a limit of detection of $0.69 \mathrm{pmol} / \mathrm{L}$.

Laboratory technicians were blinded to patient data, and test results were reported to the treating physicians. Measured biomarkers were considered abnormal when hs-cTnl was $>26.2 \mathrm{ng} /$ $\mathrm{L}$ and when copeptin was $>10 \mathrm{pmol} / \mathrm{L}^{16}$

\section{Outcome measures}

NSTEMI was defined as a typical rise and fall of hs-cTnl (a peak concentration of hs-cTnl had a relative change over 50\% if hscTnl at 0 and 2 hours were abnormal, but less than $100 \mathrm{ng} / \mathrm{L}$; or a peak concentration of hs-cTnl had a relative change over 20\% if hs-cTnl at 0 and 2 hours were abnormal and higher than $100 \mathrm{ng} /$ L) with any of the following: typical ischemic chest pain, new pathologic $\mathrm{Q}$ wave on ECG, symptomatic ST-segment depression on $\mathrm{ECG}$, and coronary stenosis on $\mathrm{CAG} .^{17}$

\section{Sample size calculation and hypothesis testing}

The study hypothesis was that the multi-marker strategy using copeptin and hs-cTnl at 0 hour was non-inferior to serial hs-cTnl measurements ( 0 and 2 hours) in terms of NPV. The prevalence of NSTEMI was estimated at 10\%. The diagnostic performance of both serial hs-cTnl measurements and the multi-marker strategy was estimated at a sensitivity of $87 \%$ and a specificity of $92 \%{ }^{18,19}$ Based on the prevalence, sensitivity, and specificity, NPV was calculated as $98.45 \%$. With the estimated NPVs of both tests and the non-inferiority margin of $4 \%$, a sample size of 235 subjects achieves $90 \%$ power at a $2.5 \%$ significance level when the probability of NSTEMI patients who were diagnosed as negative in both tests is $0 \%$ and the probability of non-NSTEMI patients who were diagnosed as negative in both tests is $82.7 \%$, which is the maximum possible value given the prevalence, sensitivity, and specificity. Accounting for a non-evaluable rate of $10 \%$, a total of 262 enrolled patients were required for the study. This sample size was derived based on the multinomial-based Wald statistic, which is a test statistic for the comparison of predictive values within a paired design that follows a chi-square distribution with one degree of freedom asymptotically under the null hypothesis. ${ }^{20}$

The sample size calculation and analysis of a non-inferiority test for the difference between two NPVs were advised by the Medical Research Collaborating Center (2017-0114).

\section{General statistics}

Categorical variables were presented as numbers with percentages, and continuous variables as median with interquartile range. A chi-square test or Fisher exact test was used to compare the categorical variables, as appropriate. The Wilcoxon rank-sum test was performed to compare the differences in continuous variables between the two groups. The diagnostic performance of biomarkers at each cutoff was evaluated in terms of sensitivity, specificity, positive predictive value (PPV), NPV, and area under the receiver operating characteristic curve (AUROC). Sensitivity, specificity, PPV, and NPV were presented as percentages with 95\% confidence intervals (Cls). A P-value less than 0.05 was considered statistically significant. Stata ver. 13.1 (StataCorp., College Station, TX, USA) was used for statistical analysis.

\section{RESULTS}

\section{Population and baseline characteristics}

A total of 316 patients with chest pain were screened during the study period. With the exclusion of 46 patients who met the exclusion criteria, 270 patients provided informed consent. After excluding seven patients who violated the protocol, withdrew 


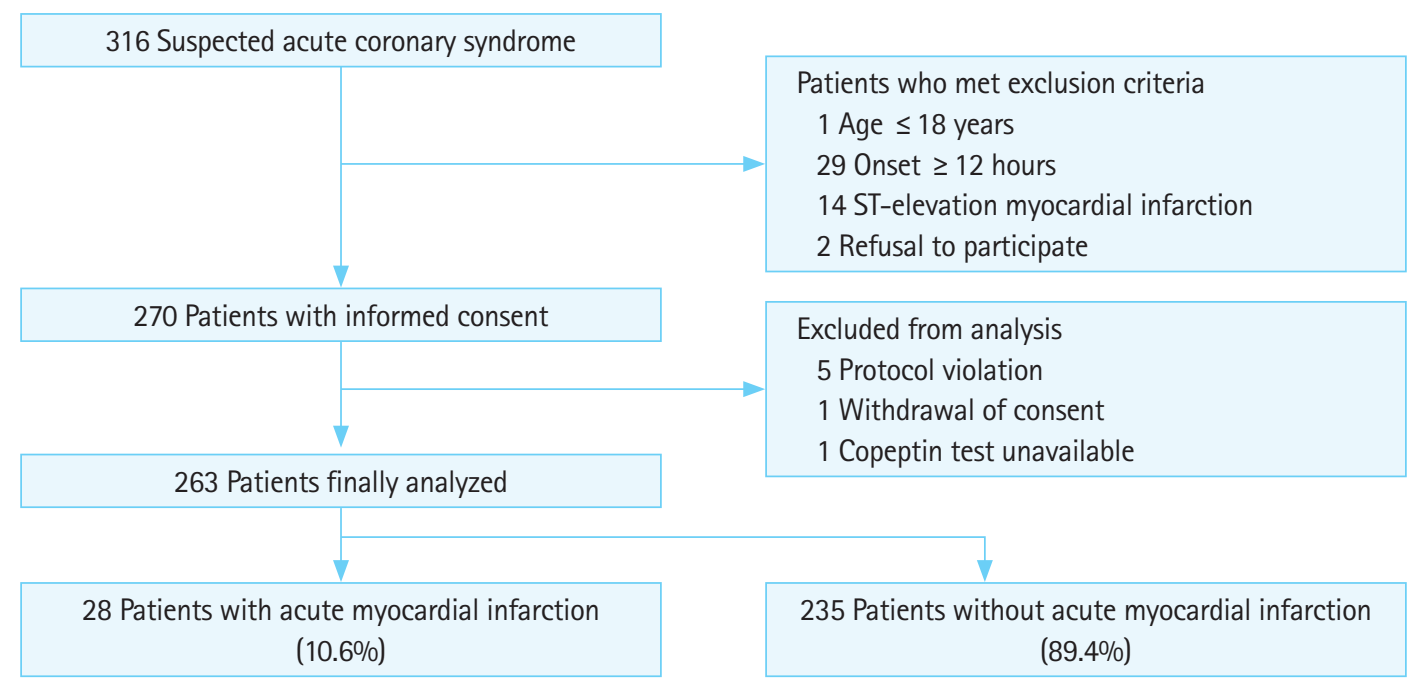

Fig. 1. Flowchart of patient enrollment.

Table 1. Characteristics of the study population

\begin{tabular}{|c|c|c|c|c|}
\hline Characteristics & All patients $(n=263)$ & NSTEMI $(n=28)$ & No NSTEMI $(\mathrm{n}=235)$ & P-value \\
\hline \multicolumn{5}{|l|}{ Baseline characteristics } \\
\hline Age (yr) & $66(57-75)$ & $68(59-76.5)$ & $66(57-75)$ & 0.383 \\
\hline Symptom-to-arrival time (min) & $124(48-269)$ & $70(27-136)$ & $148(50-280)$ & 0.025 \\
\hline$<3 \mathrm{hr}$ & $153(58.2)$ & $22(78.6)$ & $131(55.7)$ & 0.021 \\
\hline \multicolumn{5}{|l|}{ Past medical history } \\
\hline Hyperlipidemia & 89 (33.8) & $9(32.1)$ & $80(34.0)$ & 0.841 \\
\hline Family history & $44(16.7)$ & $3(10.7)$ & $41(17.5)$ & $0.591^{\mathrm{a})}$ \\
\hline Smoker & 39 (14.8) & $7(25.0)$ & $32(13.6)$ & $0.154^{\mathrm{al}}$ \\
\hline Known CAD & 79 (30.0) & $6(21.4)$ & $73(31.1)$ & 0.293 \\
\hline ASA use in past 7 days & $90(34.2)$ & $9(32.1)$ & $81(34.5)$ & 0.806 \\
\hline Copeptin $0 \mathrm{hr}$ (pmol/L) & $5.01(2.71-10.37)$ & $8.92(5.20-23.19)$ & $4.59(2.62-9.97)$ & $<0.001$ \\
\hline$>10 \mathrm{pmol} / \mathrm{L}$ & $71(27.0)$ & $13(46.4)$ & $58(24.7)$ & 0.014 \\
\hline hs-Tnl 0 hr (ng/L) & $1(1-20)$ & $525(105-3,905)$ & $1(1-1)$ & $<0.001$ \\
\hline$>26.2 \mathrm{ng} / \mathrm{L}$ & $59(22.4)$ & 27 (96.4) & $32(13.6)$ & $<0.001$ \\
\hline hs-Tnl 2 hr (ng/L) & $1(1-20)$ & $905(185-5,680)$ & $1(1-1)$ & $<0.001$ \\
\hline$>26.2 \mathrm{ng} / \mathrm{L}$ & $62(23.6)$ & $28(100)$ & $34(14.5)$ & $<0.001$ \\
\hline hs-Tnl peak (ng/L) & $1(1-60)$ & $6815(560-29,710)$ & $1(1-20)$ & $<0.001$ \\
\hline \multicolumn{5}{|l|}{ Additional testing } \\
\hline Coronary CT angiography & $86(32.7)$ & $2(7.1)$ & $84(35.7)$ & $0.001^{\mathrm{a})}$ \\
\hline Treadmill test & $5(1.9)$ & $0(0)$ & $5(2.1)$ & NA \\
\hline Cardiac scintigraphy & $7(2.7)$ & $0(0)$ & $7(3.0)$ & NA \\
\hline
\end{tabular}

Values are presented as median (interquartile range) or number (\%).

NSTEMI, non-ST-elevation myocardial infarction; CAD, coronary artery disease; ASA, aspirin; ECG, electrocardiogram; TIMI, thrombolysis in myocardial infarction; hs-Tnl, high-sensitivity troponin I; CT, computed tomography; $\mathrm{NA}$, not applicable; $\mathrm{PCl}$, percutaneous coronary intervention.

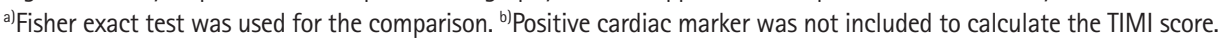


Kyung Su Kim, et al.

Table 2. Performances of copeptin, hs-Tnl, and combination of biomarkers for the diagnosis of non-ST-elevation myocardial infarction

\begin{tabular}{|c|c|c|c|c|c|}
\hline Performances & Copeptin (0 hr) & hs-Tnl (0 hr) & hs-Tnl (2 hr) & Copeptin and hs-Tnl (0 hr) & hs-Tnl ( 0 and $2 \mathrm{hr}$ ) \\
\hline True positive & 13 & 27 & 28 & 28 & 28 \\
\hline False positive & 58 & 32 & 34 & 75 & 34 \\
\hline True negative & 177 & 203 & 201 & 160 & 201 \\
\hline False negative & 15 & 1 & 0 & 0 & 0 \\
\hline Sensitivity & $46.4(27.5-66.1)$ & $96.4(81.7-99.9)$ & $100(87.7-100)$ & $100(87.7-100)$ & $100(87.7-100)$ \\
\hline Specificity & $75.3(69.3-80.7)$ & $86.4(81.3-90.5)$ & $85.5(80.4-89.8)$ & $68.1(61.7-74.0)$ & 85.5 (80.4-89.8) \\
\hline Positive predictive value & $18.3(10.1-29.3)$ & $45.8(32.7-59.2)$ & $45.2(32.5-58.3)$ & $27.2(18.9-36.8)$ & $45.2(32.5-58.3)$ \\
\hline Negative predictive value & $92.2(87.4-95.6)$ & $99.5(97.3-100)$ & $100(98.2-100)$ & $100(97.7-100)$ & $100(98.2-100)$ \\
\hline AUROC & $0.609(0.511-0.707)$ & $0.914(0.873-0.955)$ & $0.928(0.905-0.950)$ & $0.840(0.811-0.870)$ & $0.928(0.905-0.950)$ \\
\hline
\end{tabular}

Values are presented with $95 \%$ confidence intervals. Cutoff values were $10 \mathrm{pmol} / \mathrm{L}$ and $26.2 \mathrm{ng} / \mathrm{L}$ for copeptin and hs-Tnl, respectively.

hs-Tnl, high-sensitivity troponin I; AUROC, area under the receiver operating characteristic curve.

consent, and had no available copeptin test, 263 patients were finally included in the analysis (Fig. 1).

NSTEMI was diagnosed in 28 (10.7\%) patients. Patients with NSTEMI visited the ED earlier and had ischemic ECG changes more frequently (Table 1). Copeptin and hs-cTnl were significantly higher in patients with NSTEMI. CAG was performed in all patients with NSTEMI and 82 (34.9\%) patients without NSTEMI.

\section{Outcomes}

The NPVs of copeptin and hs-cTnl and serial hs-cTnl measurements for NSTEMI were 100\% (97.7\% to 100\%) and 100\% (98.2\% to $100 \%$ ), respectively (Table 2). With both NPVs of 100\%, it was not possible to calculate the multinomial-based Wald statistic. Therefore, we assumed two hypothetical situations. If we assumed that the multi-marker strategy missed one NSTEMI patient, the NPV of the multi-marker strategy was $99.4 \%(160 / 161,95 \% \mathrm{Cl}$, $96.6 \%$ to $100 \%$ ). The difference between the NPVs of the multimarker strategy and serial hs-cTnl measurements was $0.62 \%$ (95\% $\mathrm{Cl}_{1}-1.83 \%$ to $\left.0.59 \%\right)$, resulting in a P-value of $<0.001$. If we assumed that one of the true negative patients had NSTEMI, the NPV of the multi-marker strategy was 99.4\% (159/160; 95\% $\mathrm{Cl}, 96.6 \%$ to $100 \%)$. The difference between the NPVs of the multimarker strategy and serial hs-cTnl measurements was 0.63\% (95\% $\mathrm{Cl}_{1}-1.85 \%$ to $\left.0.60 \%\right)$, resulting in a P-value of $<0.001$. In both situations, the null hypothesis that the NPV of the multi-marker strategy was inferior to that of serial hs-cTnl measurements was rejected. Thus, the NPV of copeptin and hs-cTnl was not inferior to that of serial hs-cTnl measurements.

The diagnostic performance of biomarkers is described in Table 2. The sensitivity and NPV of copeptin alone were $46.4 \%(95 \% \mathrm{Cl}$, $27.5 \%$ to $66.1 \%)$ and $92.2 \%(95 \% \mathrm{Cl}, 87.4 \%$ to $95.6 \%)$, respectively. The AUROC of the multi-marker strategy was 0.840 (95\% $\mathrm{Cl}, 0.811$ to 0.870 ), which was significantly smaller than that of serial hs-cTnl measurements $(0.928 ; 95 \% \mathrm{Cl}, 0.905$ to 0.950$)(\mathrm{P}<$

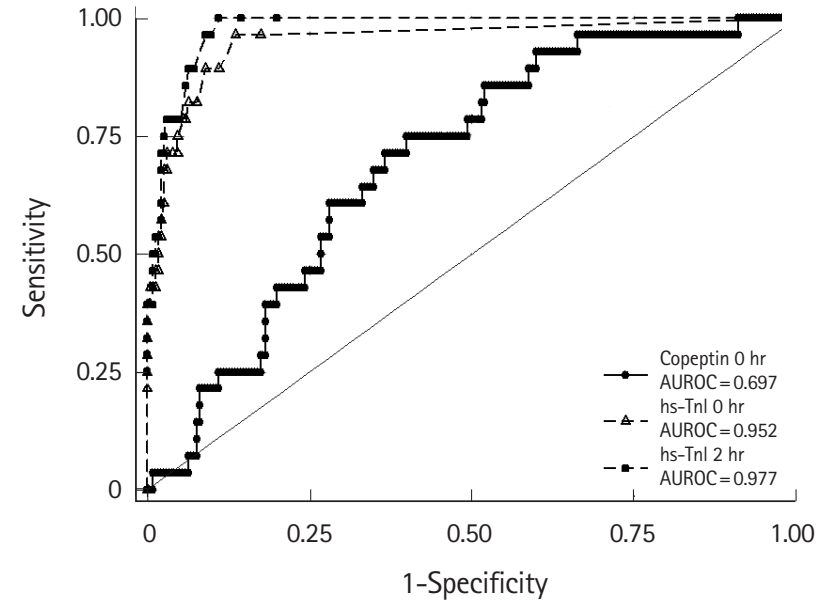

Fig. 2. Receiver operating characteristic curve of copeptin and highsensitivity troponin I (hs-cTnl) for the diagnosis of non-ST-elevation myocardial infarction. AUROC, area under the receiver operating characteristic curve.

0.001).

Fig. 2 shows the receiver operating characteristic curve of biomarkers. The AUROC of copeptin at 0 hour $(0.697 ; 95 \% \mathrm{Cl}, 0.607$ to 0.786 ) was significantly smaller than those of hs-cTnl at 0 hour (0.952; $95 \% \mathrm{Cl}, 0.909$ to $0.995 ; \mathrm{P}<0.001)$ and 2 hours ( 0.977 ; $95 \% \mathrm{Cl}, 0.962$ to $0.993 ; \mathrm{P}<0.001)$.

\section{DISCUSSION}

We compared the rapid rule-out performance between the multimarker strategy using copeptin and hs-cTnl at admission and serial hs-cTnl measurements ( 0 and 2 hours) for NSTEMI in ED patients with chest pain. The multi-marker strategy demonstrated 100\% sensitivity and 100\% NPV. The NPV of the multi-marker strategy was not inferior to that of serial hs-cTnl measurements for NSTEMI diagnosis. 
As mentioned in the Introduction section, many studies have investigated this multi-marker strategy for the rapid rule-out of NSTEMI. ${ }^{7-13}$ Because copeptin rapidly rises after endogenous stress, such as $\mathrm{AMI}{ }_{1}^{21}$ the addition of copeptin to conventional cardiac troponin assays has increased the sensitivity for the diagnosis of AMI. ${ }^{7-10,12}$ However, this additive diagnostic ability should be reevaluated in the era of high-sensitivity cardiac troponin assays. Furthermore, the multi-marker strategy using copeptin and hscTn measurement should be compared with serial hs-cTn measurements.

A study reported that the combined use of copeptin and cardiac troponin I is not inferior to serial cardiac troponin I measurement in ruling out NSTEMI. ${ }^{22}$ However, this study used mediumsensitivity troponin assays. Another study investigated the single combined testing of troponin and copeptin to rule out $\mathrm{AMI}$, and the proportion of major adverse cardiac events is not inferior in the copeptin group to that in the standard group. ${ }^{16}$ The primary outcome of interest of this study was the major adverse cardiac events during the 30-day follow-up period. A recent study was the first to compare the dual marker strategy using copeptin and high-sensitivity cardiac troponin T with other guideline-recommended fast AMI rule-out protocols and found that the dual marker strategy can be an alternative to the serial high-sensitivity cardiac troponin T-based algorithm. ${ }^{23}$

We have implemented the standardized diagnostic protocol using a 2-hour hs-cTnl measurement with TIMI score in our institution several years ago. ${ }^{14}$ Therefore, we wanted to compare the multi-marker strategy using copeptin and hs-cTnl at admission with the standard protocol. The advantage of using the multi-marker strategy is that rapid decision-making can reduce the length of stay of patients with chest pain in the ED. A previous study reported that the combined testing of copeptin and troponin could significantly reduce the length of stay by 3 hours without increasing the likelihood of major adverse cardiac events. ${ }^{16}$ In this study, we could not compare the length of stay since copeptin and serial troponin tests were performed in all patients. However, the median reported time of hs-cTnl at 2 hours was 97 minutes longer than that of the initial sample $(P<0.001$, Wilcoxon signed-rank test). According to the multi-marker strategy, $60.8 \%$ (160/263) of patients had negative results in this study. Among them, 92 patients (35.0\%) had a TIMI score of less than 2 and could be safely discharged from the single sampling strategy. Earlier decisionmaking using copeptin may lessen unnecessary stay and thus can reserve medical resources such as beds and medical personnel for more severely ill patients, especially in the overcrowded ED.

In order to achieve such an advantage, the safety of the multimarker strategy should be investigated thoroughly. Although the multi-marker strategy demonstrated excellent sensitivity and NPV, copeptin alone did not show enough discriminatory ability compared with hs-cTnl (Fig. 2). Because copeptin is not specific to cardiac injury, the specificity and PPV were lower than those of hs-cTnl. As a result, the copeptin level was increased in 58 patients without NSTEMI. Supplementary Table 1 describes the alternative diagnoses in these patients. The copeptin level was elevated in chronic kidney disease, heart failure, unstable angina, atrial fibrillation with rapid ventricular response, pneumonia, paroxysmal supraventricular tachycardia, and pulmonary embolism. In 15 patients (25.9\%), there was no obvious cause of elevated copeptin. Moreover, copeptin has inverse release kinetics compared with cTn, and the sensitivity and NPV decrease when the symptom-to-arrival time is delayed. ${ }^{24}$ In our study, the copeptin level was significantly higher in patients with NSTEMI when the symptom-to-arrival time was less than 180 minutes $(P<0.001)$. However, the copeptin level did not differ between patients with and those without NSTEMI when the symptom-to-arrival time exceeded 180 minutes $(P=0.528)$ (Supplementary Fig. 1). As a result, copeptin missed 15 patients with NSTEMI. By contrast, hscTnl missed only one patient with NSTEMI, and this patient had an elevated copeptin level. Taken together, the combined testing of copeptin and hs-cTnl demonstrated 100\% sensitivity and NPV for NSTEMI. Overall, the diagnostic value of the multi-marker strategy using copeptin and hs-cTnl for NSTEMI was primarily due to hs-cTnl.

This study has several additional limitations that need to be considered. First, this was a single-center observational study with a limited number of patients. We calculated the sample size based on a non-inferiority margin of 4\% in NPV. A larger sample size from multiple institutions is required to test the narrower margin and evaluate generalizability. Second, the diagnostic performance of biomarkers was not tested according to clinical risk scores, such as the TIMI score, because of the modest number of patients.

In conclusion, the multi-marker strategy using copeptin and hs-cTnl measurement was not inferior to serial hs-cTnl measurements in terms of NPV for NSTEMI diagnosis. Both the sensitivity and NPV of the multi-marker strategy were 100\%. However, the diagnostic performance of copeptin alone for NSTEMI was limited, especially in patients with delayed presentation. The specificity and PPV of the multi-marker strategy were lower than that of serial hs-cTnl. In the application of the multi-marker strategy using copeptin and hs-cTnl, these limitations should be considered. 


\section{CONFLICT OF INTEREST}

No potential conflict of interest relevant to this article was reported.

\section{ACKNOWLEDGMENTS}

This work was supported by ANSE Company (Seongnam, Republic of Korea; grant no. 06-2017-3910). Thermo Fisher Scientific (Seoul, Republic of Korea) supported Thermo Scientific B.R.A.H.M.S Copeptin proAVP KRYPTOR assay (B.R.A.H.M.S. GmbH, Hennigsdorf, Berlin, Germany) for this study.

\section{SUPPLEMENTARY MATERIAL}

Supplementary Table 1 and Supplementary Fig. 1 are available from: https://doi.org/10.15441/ceem.19.013.

\section{REFERENCES}

1. Mockel M, Searle J, Muller R, et al. Chief complaints in medical emergencies: do they relate to underlying disease and outcome? The Charite Emergency Medicine Study (CHARITEM). Eur J Emerg Med 2013;20:103-8.

2. Roffi M, Patrono C, Collet JP, et al. 2015 ESC guidelines for the management of acute coronary syndromes in patients presenting without persistent ST-segment elevation: Task Force for the Management of Acute Coronary Syndromes in Patients Presenting without Persistent ST-Segment Elevation of the European Society of Cardiology (ESC). Eur Heart J 2016; 37:267-315.

3. Koshimizu TA, Nakamura K, Egashira N, Hiroyama M, Nonoguchi $\mathrm{H}$, Tanoue $A$. Vasopressin V1a and V1b receptors: from molecules to physiological systems. Physiol Rev 2012;92:181364.

4. Baumann G, Dingman JF. Distribution, blood transport, and degradation of antidiuretic hormone in man. J Clin Invest 1976; 57:1109-16.

5. Struck J, Morgenthaler NG, Bergmann A. Copeptin, a stable peptide derived from the vasopressin precursor, is elevated in serum of sepsis patients. Peptides 2005;26:2500-4.

6. Vargas KG, Kassem M, Mueller C, Wojta J, Huber K. Copeptin for the early rule-out of non-ST-elevation myocardial infarction. Int J Cardiol 2016;223:797-804.

7. Ray $P_{1}$ Charpentier $S$, Chenevier-Gobeaux $C$, et al. Combined copeptin and troponin to rule out myocardial infarction in patients with chest pain and a history of coronary artery dis- ease. Am J Emerg Med 2012;30:440-8.

8. Charpentier S, Maupas-Schwalm F, Cournot M, Elbaz M, Botella JM, Lauque D. Combination of copeptin and troponin assays to rapidly rule out non-ST elevation myocardial infarction in the emergency department. Acad Emerg Med 2012; 19:517-24.

9. Charpentier S, Lepage B, Maupas-Schwalm F, et al. Copeptin improves the diagnostic performance of sensitive troponin IUltra but cannot rapidly rule out non-ST-elevation myocardial infarction at presentation to an emergency department. Ann Emerg Med 2013;61:549-58.

10. Chenevier-Gobeaux $C$, Freund Y, Claessens YE, et al. Copeptin for rapid rule out of acute myocardial infarction in emergency department. Int J Cardiol 2013;166:198-204.

11. Sebbane M, Lefebvre $S$, Kuster $N$, et al. Early rule out of acute myocardial infarction in ED patients: value of combined highsensitivity cardiac troponin T and ultrasensitive copeptin assays at admission. Am J Emerg Med 2013;31:1302-8.

12. Chenevier-Gobeaux $C$, Charpentier $S$, Meune $C$, Claessens $Y E_{\text {, }}$ Lavieuville M, Ray P. Copeptin improves the sensitivity of cardiac troponin in patients 70 years or older, but not enough to rule out myocardial infarction at emergency department presentation. Eur J Emerg Med 2017;24:142-8.

13. Stengaard C, Sorensen JT, Ladefoged SA, et al. The potential of optimizing prehospital triage of patients with suspected acute myocardial infarction using high-sensitivity cardiac troponin T and copeptin. Biomarkers 2017;22:351-60.

14. Cullen $L$, Mueller $C$, Parsonage WA, et al. Validation of highsensitivity troponin I in a 2-hour diagnostic strategy to assess 30-day outcomes in emergency department patients with possible acute coronary syndrome. J Am Coll Cardiol 2013;62: 1242-9.

15. Apple FS, Collinson PO; IFCC Task Force on Clinical Applications of Cardiac Biomarkers. Analytical characteristics of highsensitivity cardiac troponin assays. Clin Chem 2012;58:54-61.

16. Mockel M, Searle J, Hamm C, et al. Early discharge using single cardiac troponin and copeptin testing in patients with suspected acute coronary syndrome (ACS): a randomized, controlled clinical process study. Eur Heart J 2015;36:369-76.

17. Thygesen K, Mair J, Giannitsis E, et al. How to use high-sensitivity cardiac troponins in acute cardiac care. Eur Heart J 2012; 33:2252-7.

18. Reichlin $T$, Hochholzer W, Bassetti $S$, et al. Early diagnosis of myocardial infarction with sensitive cardiac troponin assays. N Engl J Med 2009;361:858-67.

19. Lipinski MJ, Escarcega RO, D'Ascenzo F, et al. A systematic review and collaborative meta-analysis to determine the incre- 
mental value of copeptin for rapid rule-out of acute myocardial infarction. Am J Cardiol 2014;113:1581-91.

20. Kosinski AS. A weighted generalized score statistic for comparison of predictive values of diagnostic tests. Stat Med 2013; 32:964-77.

21. Khan SQ, Dhillon OS, O'Brien RJ, et al. C-terminal provasopressin (copeptin) as a novel and prognostic marker in acute myocardial infarction: Leicester Acute Myocardial Infarction Peptide (LAMP) study. Circulation 2007;115:2103-10.

22. Ricci F, Di Scala R, Massacesi C, et al. Ultra-sensitive copeptin and cardiac troponin in diagnosing non-ST-segment elevation acute coronary syndromes: the COPACS Study. Am J Med 2016; 129:105-14.

23. Mueller-Hennessen $M$, Lindahl $B$, Giannitsis $E_{\text {, et al. Combined }}$ testing of copeptin and high-sensitivity cardiac troponin $\mathrm{T}$ at presentation in comparison to other algorithms for rapid ruleout of acute myocardial infarction. Int J Cardiol 2019;276: 261-7.

24. Reichlin T, Hochholzer W, Stelzig C, et al. Incremental value of copeptin for rapid rule out of acute myocardial infarction. J Am Coll Cardiol 2009;54:60-8. 
Supplementary Table 1. Alternative diagnosis of patients with positive copeptin and without non-ST-elevation myocardial infarction $(n=58)$

\begin{tabular}{lc}
\hline Alternative diagnosis & Value \\
\hline Chronic kidney disease & $15(25.9)$ \\
Heart failure & $12(20.7)$ \\
Unstable angina & $5(8.6)$ \\
Atrial fibrillation with rapid ventricular response & $5(8.6)$ \\
Pneumonia & $4(6.9)$ \\
Paroxysmal supraventricular tachycardia & $1(1.7)$ \\
Pulmonary embolism & $1(1.7)$ \\
No identifiable cause & $15(25.9)$ \\
\hline
\end{tabular}

Values are presented as number (\%).
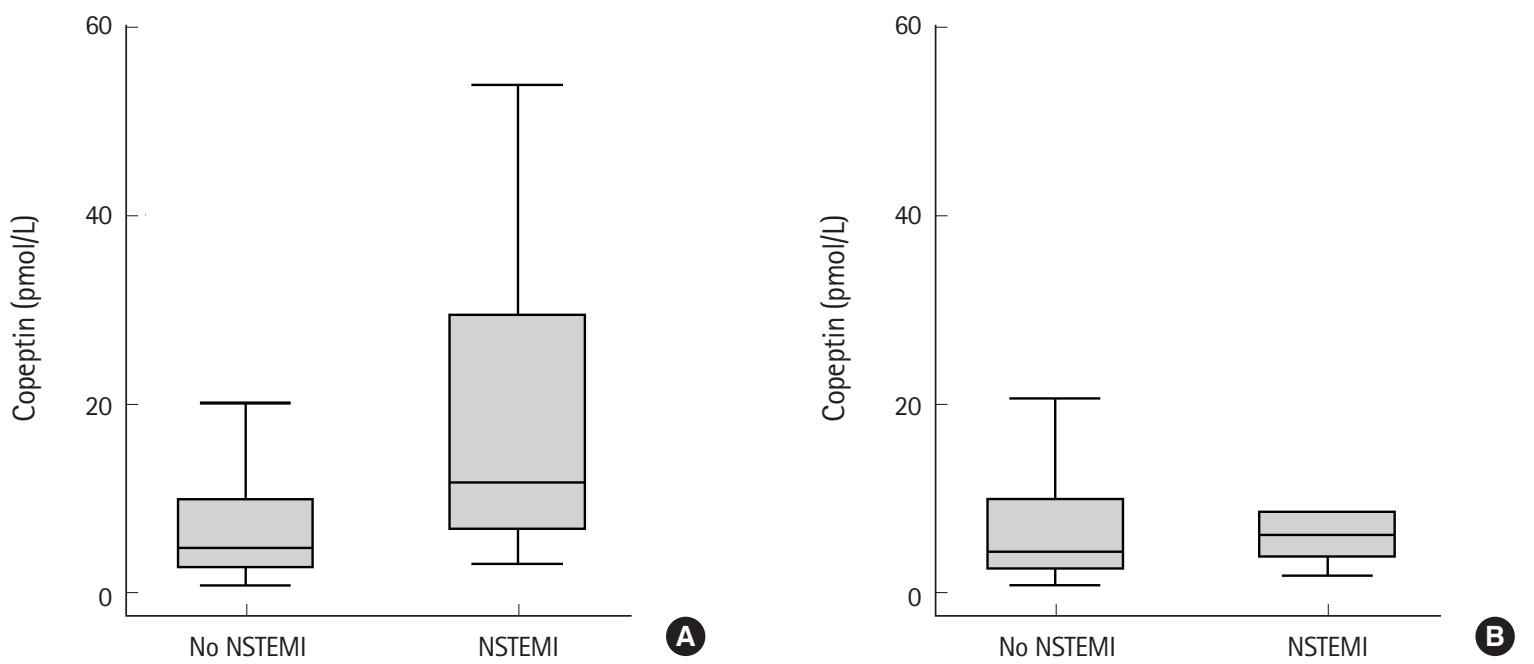

Supplementary Fig. 1. Boxplot representing copeptin levels according to diagnosis of non-ST-elevation myocardial infarction (NSTEMI). (A) Copeptin level was significantly higher in patients with NSTEMI when the symptom-to-arrival time was $<180$ minutes $(P<0.001)$. (B) Copeptin level did not differ between patients with NSTEMI and those without NSTEMI when the symptom-to-arrival time was $>180$ minutes ( $P=0.528)$. 